\title{
Efficient simulation of multimodal nonlinear propagation in step-index fibers
}

\author{
Lægsgaard, Jesper
}

Published in:

Journal of the Optical Society of America B-optical Physics

Link to article, DOI:

10.1364/JOSAB.34.002266

Publication date:

2017

Document Version

Publisher's PDF, also known as Version of record

Link back to DTU Orbit

Citation (APA):

Lægsgaard, J. (2017). Efficient simulation of multimodal nonlinear propagation in step-index fibers. Journal of the Optical Society of America B-optical Physics, 34(10), 2266-2273. https://doi.org/10.1364/JOSAB.34.002266

\section{General rights}

Copyright and moral rights for the publications made accessible in the public portal are retained by the authors and/or other copyright owners and it is a condition of accessing publications that users recognise and abide by the legal requirements associated with these rights.

- Users may download and print one copy of any publication from the public portal for the purpose of private study or research.

- You may not further distribute the material or use it for any profit-making activity or commercial gain

- You may freely distribute the URL identifying the publication in the public portal

If you believe that this document breaches copyright please contact us providing details, and we will remove access to the work immediately and investigate your claim. 


\title{
Efficient simulation of multimodal nonlinear propagation in step-index fibers
}

\author{
JESPER L/EGSGAARD (i) \\ DTU Fotonik, Department of Photonics Engineering, Technical University of Denmark, Orsteds Plads 343 , \\ DK-2800 Kongens Lyngby, Denmark (jlag@fotonik.dtu.dk)
}

Received 22 June 2017; revised 5 September 2017; accepted 6 September 2017; posted 7 September 2017 (Doc. ID 300785); published 28 September 2017

\begin{abstract}
A numerical approach to nonlinear propagation in waveguides based on real-space Gaussian quadrature integration of the nonlinear polarization during propagation is investigated and compared with the more conventional approach based on expressing the nonlinear polarization by a sum of mode overlap integrals. Using the step-index fiber geometry as an example, it is shown that the Gaussian quadrature approach scales linearly or at most quadratically with the number of guided modes and that it can account for mode profile dispersion without additional computational overhead. These properties make it superior for multimode nonlinear simulations extending over wide frequency ranges. (๑) 2017 Optical Society of America
\end{abstract}

OCIS codes: (060.4370) Nonlinear optics, fibers; (060.5530) Pulse propagation and temporal solitons; (190.0190) Nonlinear optics.

https://doi.org/10.1364/JOSAB.34.002266

\section{INTRODUCTION}

Nonlinear propagation in multimoded fibers is currently of high interest in connection with both space-division multiplexing in telecommunication and nonlinear frequency conversion. In telecommunication, the use of different fiber modes to carry different data streams is envisioned to boost the signal transmitting capacity of single fiber cores $[1,2]$. Because of the high total field intensities obtained, and long propagation lengths, it is crucial to understand intermodal nonlinear interactions in such applications [3]. In addition, multimode waveguides have been shown to open up new opportunities for frequency conversion by nonlinear effects. Interactions between different guided modes can lead to phase-matched four-wave mixing over wide frequency ranges and with pump wavelengths in the normal-dispersion regime of the fibers [4-6]. Pumping large-core multimode fibers with a parabolic index variation to minimize walkoff between guided modes has been shown to enable complex intermodal nonlinear interactions, in particular enabling short-wavelength dispersive-wave generation from soliton collapse and spatiotemporal soliton oscillations at elevated pulse energies $[7,8]$, and sideband formation from geometric parametric instabilities [9]. Also, in the emerging low-loss hollow-core fibers guiding by antiresonant effects, intermodal nonlinearities can be important for the highly interesting frequency-conversion processes enabled by these waveguides [10]. Because of these emerging applications and the continuing development of efficient methods for manipulating input pulse profiles, it is of great importance to devise efficient numerical methods for describing nonlinear propagation of high-power ultrafast pulses distributed over many spatial modes, and possibly spanning a wide frequency range during propagation. However, multimode formulations of the generalized nonlinear Schrödinger equation based on an expansion of the nonlinear polarization via nonlinear mode overlap integrals scale badly with the number of modes in terms of computational efficiency [11]. In addition, an accurate and wellcontrolled treatment of mode profile variations with frequency is not straightforward with such methods $[12,13]$. The purpose of this paper is to investigate an alternative approach using realspace integration of the spatial nonlinear polarization on a Gaussian quadrature (GQ) grid during propagation [14], in the context of multimodal propagation in optical fibers. A similar method was recently used by Tani $e t$ al. to treat ionization effects in gas-filled hollow-core fibers [10]. The approach is tested by studying generation of dispersive waves, commonly denoted "Cherenkov radiation" (CR) [15] in multimode step-index fibers pumped by ultrafast high-power pulses. The GQ is shown to have superior scaling properties when the number of guided modes is increased, to handle mode profile dispersion efficiently, and to be comparable in numerical complexity to an approach based on mode overlap integrals already for a test case with six fiber modes and neglect of mode profile dispersion. Although the application testing is here limited to CR generation in short fibers, the method described is expected to be applicable to and relevant for a wide range of contemporary nonlinear propagation problems. 


\section{FORMAL THEORY}

\section{A. Propagation Equations}

Expanding the propagating electric field in the fiber core as

$$
\mathbf{E}(\mathbf{r}, t)=\frac{1}{2 \pi} \sum_{m} \int \mathrm{d} \omega \tilde{G}_{m}(z, \omega) \mathbf{e}_{m}\left(\mathbf{r}_{\perp}, \omega\right) e^{\left[i\left(\beta_{m}(\omega) z-\omega t\right)\right]},
$$

with a corresponding expansion for the magnetic field, the nonlinear propagation equation may be written as $[12,14]$

$$
\begin{aligned}
\frac{\partial \tilde{G}_{m}(z, \omega)}{\partial z}= & \frac{\varepsilon_{0} \chi^{(3)} 2 \pi i \omega}{2 N_{m}(\omega)} e^{-i \beta_{m}(\omega) z} \int \mathrm{d} t e^{i \omega t} \int \mathrm{d} \mathbf{r}_{\perp} \\
& \times \mathbf{e}_{m}^{*}\left(\mathbf{r}_{\perp}, \omega\right) \cdot \mathbf{E}(\mathbf{r}, t) \int \mathrm{d} t^{\prime} R\left(t-t^{\prime}\right)\left|\mathbf{E}\left(\mathbf{r}, t^{\prime}\right)\right|^{2} .
\end{aligned}
$$

In these equations, $\mathbf{e}_{m}\left(\mathbf{r}_{\perp}, \omega\right), \mathbf{h}_{m}\left(\mathbf{r}_{\perp}, \omega\right)$ are the transverse field distributions of the guided modes, indexed by $m$, and $\beta_{m}(\omega)$ are their frequency-dependent propagation constants. $N_{m}(\omega)$ is a normalization parameter given by

$$
\int \mathrm{d} \mathbf{r}_{\perp}\left[\mathbf{e}_{m} \times \mathbf{h}_{n}^{*}+\mathbf{e}_{n}^{*} \times \mathbf{h}_{m}\right]=2 N_{m}(\omega) \delta_{m n}
$$

and $R(t)$ is the nonlinear response function, which is a combination of a delta-function Kerr term and a delayed Raman term. In deriving Eq. (2), cross-polarization Raman scattering has been neglected, and the nonlinear susceptibility $\chi^{(3)}$ has been assumed isotropic and position-independent. A common way to approach Eq. (2) or similar formulations is to insert the expansion in Eq. (1) for the electric field and perform the transverse integrations $[10,11,16,17]$. This overlap integral (OI) approach leads to the expressions

$$
\begin{aligned}
& \frac{\partial \tilde{G}_{m}(z, \omega)}{\partial z}= \frac{i \omega \varepsilon_{0}}{4 \pi N_{m}(\omega)} e^{-i \beta_{m}(\omega) z} \sum_{n q p} \int \mathrm{d} \omega_{1} \mathrm{~d} \omega_{2} \\
& \times G_{n}\left(z, \omega_{1}\right) G_{q}^{*}\left(z, \omega_{1}+\omega_{2}-\omega\right) G_{p}\left(z, \omega_{2}\right) \\
& \times R\left(\omega-\omega_{1}\right) K_{m n q p}\left(\omega, \omega_{1}, \omega_{2}\right) \\
& G_{m}(z, \omega)=\tilde{G}_{m}(z, \omega) e^{i \beta_{m}(\omega) z} \\
& K_{m n q p}\left(\omega, \omega_{1}, \omega_{2}\right) \equiv \chi^{(3)} \int \mathrm{d} \mathbf{r}_{\perp} \mathbf{e}_{m}^{*}\left(\mathbf{r}_{\perp}, \omega\right) \\
& \cdot \mathbf{e}_{n}\left(\mathbf{r}_{\perp}, \omega_{1}\right) \mathbf{e}_{q}^{*}\left(\mathbf{r}_{\perp}, \omega_{1}+\omega_{2}-\omega\right) \cdot \mathbf{e}_{p}\left(\mathbf{r}_{\perp}, \omega_{2}\right) .
\end{aligned}
$$

Numerically, the double frequency integral in Eq. (4) is very demanding when a general frequency dependence of the modes is allowed. Assuming frequency-independent $\mathbf{e}_{m}$, one obtains the more tractable expression

$$
\begin{gathered}
\frac{\partial \tilde{G}_{m}(z, \omega)}{\partial z}=\frac{i \omega \varepsilon_{0}}{4 \pi N_{m}(\omega)} e^{-i \beta_{m}(\omega) z} \int \mathrm{d} t e^{i \omega t}, \\
\sum_{n q p} K_{m n q p} G_{m}(z, t) \int \mathrm{d} t^{\prime} G_{q}^{*}\left(z, t^{\prime}\right) G_{p}\left(z, t^{\prime}\right) R\left(t-t^{\prime}\right) \\
G_{m}(z, t)=\frac{1}{2 \pi} \int_{-\infty}^{\infty} \mathrm{d} \omega G_{m}(z, \omega) e^{-i \omega t} .
\end{gathered}
$$

The overlap integrals $K_{m n q p}$ are given by Eq. (6) with all frequencies set equal to some chosen base frequency $\omega_{0}$. With $M$ modes in the field expansion, and a Fourier grid with $N$ points, evaluation of the RHS of Eq. (7) requires $2 M$ complex fast Fourier transform (FFT) operations to transform the $G_{m}$ from frequency to time and back, as well as $2 M^{2}$ real FFTs to evaluate the convolution integrals in time. The numerical complexity of the FFT operations is $O(N \log N)$. In addition, multiplication of the resulting functions adds an overhead of $O\left(M^{4} N\right)$. This unfavorable scaling of computational effort with $M$ is a major impediment for simulation of multimoded nonlinear propagation.

An alternative approach to Eq. (2) is to avoid the modal overlap integrals and evaluate the spatial integral "on the fly," as

$$
\frac{\partial \tilde{G}_{m}(z, \omega)}{\partial z}=\frac{i \omega \pi \varepsilon_{0} \chi^{(3)}}{N_{m}(\omega)} e^{-i \beta_{m}(\omega) z} \int \mathrm{d} t e^{i \omega t},
$$

$$
\begin{aligned}
& \sum_{k=1}^{P} w_{k} \mathbf{e}_{m}^{*}\left(\mathbf{r}_{\perp k}\right) \cdot \mathbf{E}\left(\mathbf{r}_{\perp k}, z, t\right) \int \mathrm{d} t^{\prime}\left|\mathbf{E}\left(\mathbf{r}_{\perp k}, z, t^{\prime}\right)\right|^{2} R\left(t-t^{\prime}\right) \\
& \mathbf{E}\left(\mathbf{r}_{\perp k}, z, t\right)=\sum_{m} G_{m}(z, t) \mathbf{e}_{m}\left(\mathbf{r}_{\perp k}\right) .
\end{aligned}
$$

Here $\mathbf{r}_{\perp k}$ are a set of $P$ suitably chosen integration points, $w_{k}$ are the corresponding integration weights, and the frequency dependence of the fields is still neglected. The numerical complexity can now be estimated as $2 M$ complex FFTs, $2 P$ real FFTs, as well as $O(P M)$ order- $N$ operations multiplying the fields. The central question is therefore how large $P$ must be to ensure an adequate accuracy of the nonlinear simulation.

The frequency dependence of the modes is straightforwardly taken into account by modifying Eq. (10) to

$$
\mathbf{E}\left(\mathbf{r}_{\perp k}, z, t\right)=\frac{1}{2 \pi} \int \mathrm{d} \omega e^{-i \omega t} \sum_{m} G_{m}(z, \omega) \mathbf{e}_{m}\left(\mathbf{r}_{\perp k}, \omega\right) .
$$

Indeed, the GQ approach has previously been used by Kolesik and Moloney to accurately describe the frequency dispersion of a single mode [14]. Note that when using Eq. (11) instead of Eq. (10), $P$ complex FFT operations are needed to establish $\mathbf{E}\left(\mathbf{r}_{\perp k}, z, t\right)$. Similarly, the FFT back into the frequency domain must be done at each point on the GQ grid rather than after the spatial integration. The total numerical complexity then becomes $2 P$ complex FFTs, as well as $2 P$ real FFTs, and $O(P M)$ order- $N$ operations.

It may also be noted that if delayed (Raman) response can be neglected, the time convolution integral is replaced by a simple product, and the real FFTs are not needed. If mode profile dispersion is also neglected, the complexity of the OI and GQ methods scale as $O(M N \log N)$ and $O(2 P N \log N)$ if the FFTs dominate. However, the penalty for including mode profile dispersion is now large, going from $O(M N \log N)$ to $O(P N \log N)$ because, as will be shown in Section 3,P is typically an order of magnitude larger than $M$ for a stepindex fiber. For the operations linear in $N$, the complexity still scales as $O\left(M^{4} N\right)$ for the OI method and as $O(M P N)$ for the GQ method.

In this paper, linearly polarized scalar modes will be considered for simplicity. Inclusion of different polarization states in the GQ approach is, however, straightforward. It will double the number of modes, whereas the number of FFT operations to be performed per gridpoint will be doubled if only transverse polarization components are considered, or tripled 
if longitudinal polarization components need to be included in the total electric field, as may be relevant in some high-index contrast waveguides.

Equation (9) and its counterpart with frequency-dependent eigenmodes conserve the classical photon number in the absence of propagation losses. Taking $N_{m}(\omega)=1$ for all modes for simplicity, the photon number $N_{\text {ph }}$ may be written as

$$
N_{\mathrm{ph}}=\sum_{m} \int \mathrm{d} \omega \frac{\left|\tilde{G}_{m}(z, \omega)\right|^{2}}{\hbar \omega}
$$

and its $z$-derivative as

$$
\begin{aligned}
& \frac{d N_{\mathrm{ph}}}{d z}=\sum_{m} \int \mathrm{d} \omega \frac{2 \operatorname{Re}\left[\tilde{G}_{m}^{*}(z, \omega) \frac{\partial \tilde{G}_{m}(z, \omega)}{\partial z}\right]}{\hbar \omega} \\
& =\operatorname{Re}\left[\sum_{m} \int \mathrm{d} \omega \tilde{G}_{m}^{*}(z, \omega) \frac{i 2 \pi \varepsilon_{0} \chi^{(3)}}{\hbar} e^{-i \beta_{m}(\omega) z}\right. \\
& \quad \times \int \mathrm{d} t e^{i \omega t} \sum_{k=1}^{P} w_{k} \mathbf{e}_{m}^{*}\left(\mathbf{r}_{\perp k}, \omega\right) \cdot \mathbf{E}\left(\mathbf{r}_{\perp k}, z, t\right) \\
& \left.\quad \times \int \mathrm{d} t^{\prime}\left|\mathbf{E}\left(\mathbf{r}_{\perp k}, z, t^{\prime}\right)\right|^{2} R\left(t-t^{\prime}\right)\right] .
\end{aligned}
$$

Here $\hbar$ denotes Planck's constant. Using Eqs. (5) and (11), this expression can be rewritten as

$$
\begin{aligned}
\frac{d N_{\mathrm{ph}}}{d z}= & \operatorname{Re}\left[\frac{i(2 \pi)^{2} \varepsilon_{0} \chi^{(3)}}{\hbar} \int \mathrm{d} t \sum_{k=1}^{P} w_{k}\left|\mathbf{E}\left(\mathbf{r}_{\perp k}, z, t\right)\right|^{2}\right. \\
& \left.\times \int \mathrm{d} t^{\prime}\left|\mathbf{E}\left(\mathbf{r}_{\perp k}, z, t^{\prime}\right)\right|^{2} R\left(t-t^{\prime}\right)\right]=0,
\end{aligned}
$$

since $\chi^{(3)}$ and $R(t)$ are real functions in the absence of nonlinear losses. Eq. (15) shows that photon number conservation is a generic feature of the propagation equation and does not depend on the GQ integration accuracy.

\section{B. Analysis of Parabolic-Index Fiber}

The numerical complexity of Eqs. (9) and (10) may be analyzed precisely for the special case of scalar modes in an ideal parabolic-index fiber with a circularly symmetric index distribution of the form $n(r)=n_{0} \sqrt{1-\left(\frac{r}{r_{0}}\right)^{2}}$. Linearly polarized scalar modes in such a fiber can be written as

$$
\mathbf{e}_{k l}\left(\mathbf{r}_{\perp}, \omega\right)=\hat{\mathbf{p}} e^{-a x^{2}} p_{k-1}(x) e^{-a y^{2}} p_{l-1}(y) ; \quad a=\frac{n_{0} \omega}{2 c r_{0}},
$$

where $p_{k}$ denotes a polynomial of order $k$ and $\hat{\mathbf{p}}$ is a unit polarization vector. In the following, $\hat{\mathbf{p}}=\hat{\mathbf{x}}$ will be assumed for all modes. Evaluating the RHS of Eq. (9) now amounts to radially integrating a sum of products of four mode profiles. Since the exponential prefactor is the same for all modes, any such product can be written as $e^{-4 a\left(x^{2}+y^{2}\right)}$ multiplied by polynomials in $x$ and $y$ of degree $\leq 4\left(l_{\max }-1\right)$, where $l_{\max }$ is the highest possible value of the $k, l$ indices of the eigenmodes. The technique of GQ allows for determination of a set of $2\left(l_{\max }-1\right)$ integration points $r_{k}$ and weights $w_{k}$ such that

$$
\int_{-\infty}^{\infty} \mathrm{d} x e^{-4 a x^{2}} p_{m}(x)=\sum_{k=1}^{2\left(l_{\max }-1\right)} w_{k} p_{m}\left(x_{k}\right)
$$

for all $p_{m}$ with $m \leq 4\left(l_{\max }-1\right)$ [18]. In this way, the RHS of Eq. (9) can be evaluated as

$$
\begin{gathered}
\frac{i \omega \varepsilon_{0}}{4 \pi N_{m}(\omega)} e^{-i \beta_{m}(\omega) z} \int \mathrm{d} t e^{i \omega t} \sum_{p=1}^{2 l_{\text {max }}-1} \sum_{q=1}^{2 l_{\text {max }}-1} w_{p q} \mathbf{e}_{m}\left(\mathbf{r}_{\perp p q}\right) \\
\mathbf{E}\left(\mathbf{r}_{\perp p q}, z, t\right) \int \mathrm{d} t^{\prime}\left|\mathbf{E}\left(\mathbf{r}_{\perp p q}, z, t^{\prime}\right)\right|^{2} R\left(t-t^{\prime}\right) \\
\mathbf{r}_{\perp p q}=\left(x_{p}, x_{q}\right) ; \quad w_{p q}=w_{p} w_{q} .
\end{gathered}
$$

Thus, $P=4\left(l_{\max }-1\right)^{2}$, and the total number of guided modes that can be treated accurately is $M=l_{\max }^{2}$. This means that $P$ scales linearly with $M$, so that the numerical complexity of the nonlinear propagation becomes $O(M N \log N)$ if FFTs dominate, and $O\left(M^{2} N\right)$ if order- $N$ operations dominate. In a problem with many modes, this is a huge benefit compared with the $O\left(M^{2} N \log N\right)$ or $O\left(M^{4} N\right)$ complexity of the OI approach.

\section{Step-Index Fiber}

An important question is whether the linear scaling of $P$ with $M$ is a special feature of the parabolic index distribution, due to the particular mathematical properties of its eigenmodes, or whether it holds more generally. The most important example structure in standard fiber optics is the step-index fiber. The eigenmodes of this fiber cannot be separated in $x$ - and $y$-dependent functions as the eigenmodes of the parabolicindex fibers. However, like the eigenmodes of any index distribution with cylindrical symmetry, they can be split into products of radial and angular functions in a cylindrical coordinate system:

$$
\Psi_{m l}\left(\mathbf{r}_{\perp}\right)=R_{m l}(r) f_{m}(\phi),
$$

where $r$ is the distance from the fiber center, and $\phi$ is, say, the angle between $\mathbf{r}_{\perp}$ and the cartesian $x$-axis. $f_{m}$ may be chosen as either complex exponentials, $f_{m}(\phi)=e^{ \pm i m \phi}$, or trigonometric functions, $f_{m}(\phi)=\cos (m \phi)$ or $f_{m}(\phi)=\sin (m \phi)$. Clearly, $m$ must be an integer to make $f_{m}(\phi) 2 \pi$-periodic. For each $m$, there may be several guided eigenmodes, which are counted by the index $l$.

In line with these modal properties, a radial-angular integration grid is chosen, with points given by

$$
\mathbf{r}_{\perp p q}=r_{p}\left(\cos \left(2 \pi \frac{q-1}{4 M+1}\right), \sin \left(2 \pi \frac{q-1}{4 M+1}\right)\right) .
$$

Here, $q$ runs from 1 to $4 m_{\max }+1$, and $m_{\max }$ is the maximal value of $m$ occurring among the guided modes of interest. This angular grid is sufficient to integrate any product of four $f_{m}$ functions of the kind described previously. The radial grid is again chosen as GQ points, in this case determined to exactly integrate low-order polynomials (Gauss-Legendre integration [18]) over an interval from $r=0$ to $r=r_{s}$, where the outer radius $r_{s}$ is a free parameter to be optimized. If $r_{s}$ is too small, integration of poorly confined modes will be inaccurate. If it is too large, an excessive number of radial GQ points will be required for well-confined modes. 
Since $R_{m l}(r)$ for a step-index fiber cannot be written as a finite polynomial, the number of $r_{p}$ points required cannot be determined from an exact mathematical analysis. This problem must instead be analyzed by numerical calculations. One option is to do direct convergence checks for a specific nonlinear problem by gradually increasing the number of $r_{p}$ points, but a convenient proxy also exists. Consider the overlap integral

$$
\int \mathrm{d} \mathbf{r}_{\perp} \Psi_{m l}^{4}\left(\mathbf{r}_{\perp}\right)
$$

which equals the inverse of the classical effective area of the $m l$ mode if $\Psi_{m l}$ is properly normalized. If $\Psi_{m l}$ is the mode with the most complicated radial structure, this product must be expected to require the largest number of radial GQ points. Therefore, the accuracy of the GQ integration can be estimated by comparing effective areas calculated on a specific GQ grid with the exact values calculated on a fine grid, and this analysis may be performed ahead of any nonlinear simulations.

\section{NUMERICAL RESULTS}

\section{A. Linear Scaling of $\boldsymbol{P}$ versus $\boldsymbol{M}$}

The most important question is to determine whether the total number of radial-angular grid points $P$ needed for an accurate nonlinear simulation scales linearly with the number of modes $M$, as was the case for a parabolic-index fiber. This question is here addressed in the following way: for a step-index fiber with core radius $r_{c}=7 \mu \mathrm{m}$ and index difference $\Delta n=0.01$ between core and cladding, the guided-mode fields were determined over a range of wavelengths. Although analytical solutions do exist for the step-index fiber geometry, the fields were calculated using a radial finite-difference approach, which can be readily generalized to other cylindrically symmetric fiber structures and which treats guided and non-guided modes on the same footing. A zero-field boundary condition was applied at an outer radius of $62.5 \mu \mathrm{m}$, meaning that even non-guided modes would be confined within this radius. A more realistic description of a coated fiber might be obtained by replacing this simple boundary condition with an absorbing cladding layer, but this was not investigated here. Guided modes were determined simply as those which that effective index $n_{\text {eff }}=c \beta / \omega$ larger than the cladding index. As the wavelength gets shorter, the number of guided modes increases. For each wavelength, GQ grids were determined as described earlier, with $m_{\max }$ being the highest $m$-value occurring among the guided modes. The number of radial meshpoints, $n$, was increased until all effective areas calculated using GQ integration were accurate within some specified criterion and for a particular value of the outer integration radius $r_{s}$. The results are shown in Fig. 1. Two $r_{s}$ values of $1.3 r_{c}$ and $2 r_{c}$ have been considered. For each $r_{s}$ value, the maximal relative deviation of effective areas, $\Delta A_{\text {eff }}$, is required to be less than $10 \%, 5 \%$, or $1 \%$ for all guided modes, and the data points show the values of $P=n\left(4 m_{\max }+1\right)$ needed to match these requirements. $P$ is plotted as a function of $M$, which means there may be several results for each $M$, because some of the investigated wavelengths had the same number of guided modes. For a few wavelengths, it was not possible to reduce $\Delta A_{\text {eff }}$ to all required thresholds. To make these cases stand out in the plot, $P$ was set to zero, so that

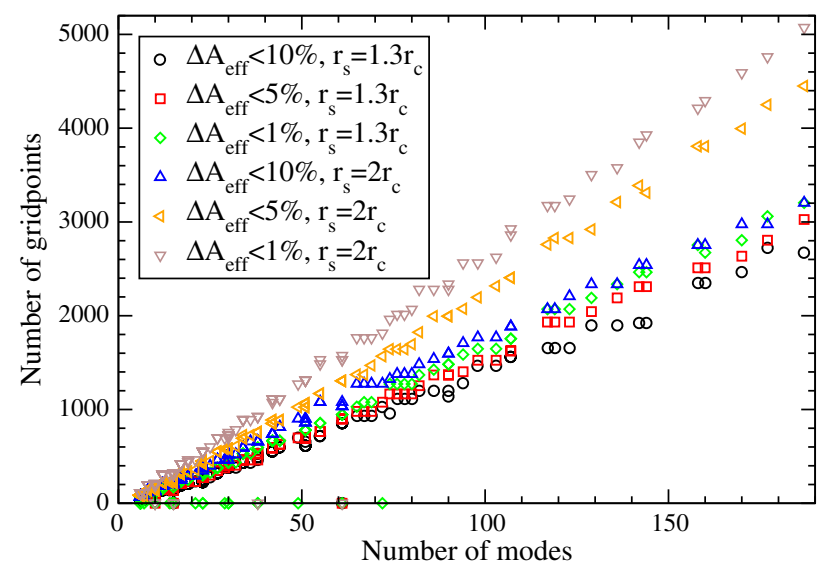

Fig. 1. Total number of GQ integration points required to calculate effective areas of all guided modes in a step-index fiber within a specified relative accuracy, versus the number of guided modes. Results are shown for two values of the outer integration boundary, $r_{s}=1.3 r_{c}$ and $r_{s}=2 r_{c}$. Zero values indicate that some effective areas could not be converged within the specified accuracy.

the data points fall on the $x$-axis. This problem occurs for poorly confined modes close to cutoff, when $r_{s}$ is not large enough for a proper description of the mode profile. As $r_{s}$ is increased from $1.3 r_{c}$ to $2 r_{c}$, the incidence of these points is strongly reduced. On the other hand, an increased value of $r_{s}$ leads to a higher slope of the $P$ versus $M$ curves. Nevertheless, a linear scaling of $P$ with $M$ is apparent in all cases. It can therefore be expected that the numerical complexity of nonlinear simulations using the GQ approach will scale as $O(M N \log N)$ or $O\left(M^{2} N\right)$ depending on which part of the calculation dominates. Compared with the mode overlap approach, this saves 1 to 2 orders of $M$, which will make a huge difference as $M$ becomes large.

\section{B. Comparison between Mode Overlap and GQ Approach}

Having established the superior scaling properties of the GQ approach, the next question is to estimate at what value of $M$ the two approaches are equal. Since more than one GQ point will be needed to correctly integrate the fundamental mode of a singlemode fiber, clearly the mode overlap approach is superior in this case. The next relevant case for a step-index fiber is three guided modes above the $\mathrm{LP}_{11}$ cutoff (not counting polarization states), and the following is six guided modes above the $\mathrm{LP}_{21} / \mathrm{LP}_{02}$ cutoff. The latter case will be considered in this subsection.

The fiber geometry discussed in the previous subsection is considered. As a test case, soliton compression and breakup of femtosecond ( $\mathrm{fs}$ ) pulses at $1.55 \mu \mathrm{m}$ wavelength is investigated. This is highly relevant for recent frequency-conversion experiments and critically tests the model's ability to describe strong nonlinear processes over a broad frequency range. On the other hand, because of the short propagation lengths, it is still a numerically tractable problem that allows for careful convergence checks with a reasonable computational effort.

To further simplify the problem, mode profile dispersion is initially neglected, with guided-mode profiles being calculated 
at the input wavelength of $1.55 \mu \mathrm{m}$. In that case, the mode overlap method is exact, within the overall approximations made in deriving Eq. (2), and may serve as a benchmark for testing GQ grids of different sizes. In Fig. 2, the output spectrum of a test calculation using the mode overlap approach is shown. A Gaussian input pulse having a peak power of $1 \mathrm{MW}$ and a full width at half-maximum (FWHM) of $50 \mathrm{fs}$ is distributed equally, and with equal phase, over the six guided modes and propagated over a distance of $2.1 \mathrm{~cm}$. The calculations were done on a Fourier grid with 2048 points spanning frequencies from 100 to $750 \mathrm{THz}$ (3.0-0.4 $\mu \mathrm{m}$ wavelengths). As can be seen from the output spectrum, this is sufficient to ensure nonlinear compression and breakup with Raman redshifted solitons and CR emission around $800 \mathrm{~nm}$. Because of the technical focus of this paper, the detailed nonlinear dynamics leading to the final spectrum will not be discussed here. An important point is, however, that both the short-wavelength $\mathrm{CR}$ and the spectral weight appearing beyond $2 \mu \mathrm{m}$ is mainly in the $\mathrm{LP}_{21}$ modes.

To validate the GQ approach, simulations are performed with a varying number of radial gridpoints, $n$, whereas the number of angular gridpoints is kept fixed at nine, which allows for exact integration in this coordinate. In Fig. 3, the total spectral density, summed over all modes, is shown for GQ calculations with $n=5$ and $n=6$, compared with the result of an OI calculation. It can be seen that the $n=5$ result deviates significantly from the OI result, whereas the differences are slight for the $n=6$ case. In Fig. 4, the integral of the absolute value of the deviation in total spectral density between the OI and GQ methods is plotted, normalized to the integral of total spectral density for the OI calculation. Curves are given for GQ calculations with $r_{s}=1.3 r_{c}$ and $r_{s}=2 r_{c}$, respectively. For the low value of $r_{s}$, a deviation slightly below $1 \%$ can be obtained. For $n>9$, there is no significant improvement. For $r_{s}=2 r_{c}$, the deviation could be driven below the per mill level for $n=40$ (not shown), which indicates that the remaining error

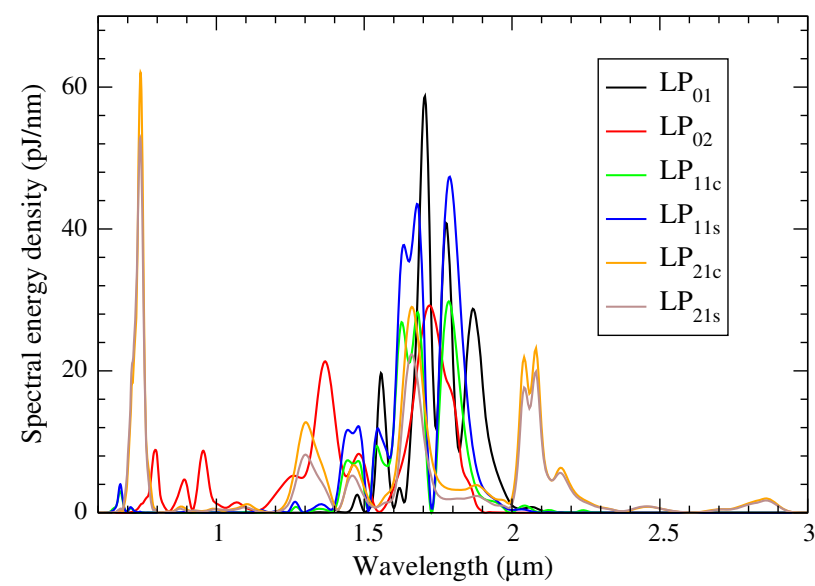

Fig. 2. Spectral densities for the six modes of a fiber with core radius $r_{c}=7 \mu \mathrm{m}$, core-cladding index difference $\Delta n=0.01$, after propagation over $2.1 \mathrm{~cm}$ of a $50 \mathrm{fs}$ input pulse with $1 \mathrm{MW}$ peak power, evenly distributed over the six modes, with fixed linear polarization. Index labels $c$ and $s$ on the $\mathrm{LP}_{m 1}$ modes with $m>0$ indicate modes with angular dependence of $\cos (m \phi), \sin (m \phi)$, respectively. The results were found using the OI method with mode profiles calculated at a wavelength of $1.55 \mu \mathrm{m}$.

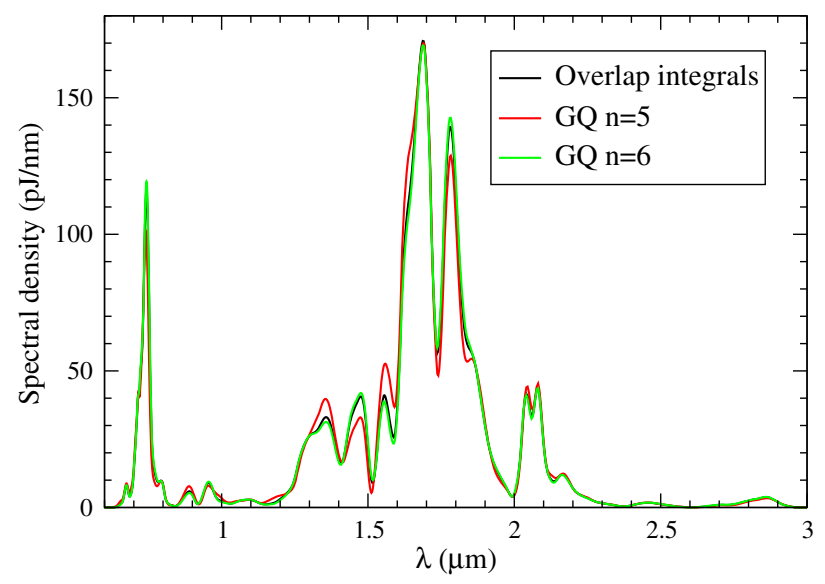

Fig. 3. Total spectral densities, summed over all modes, for the OI calculation shown in Fig. 2, as well as corresponding GQ calculations with $r_{s}=1.3 r_{c}, n=5-6$.

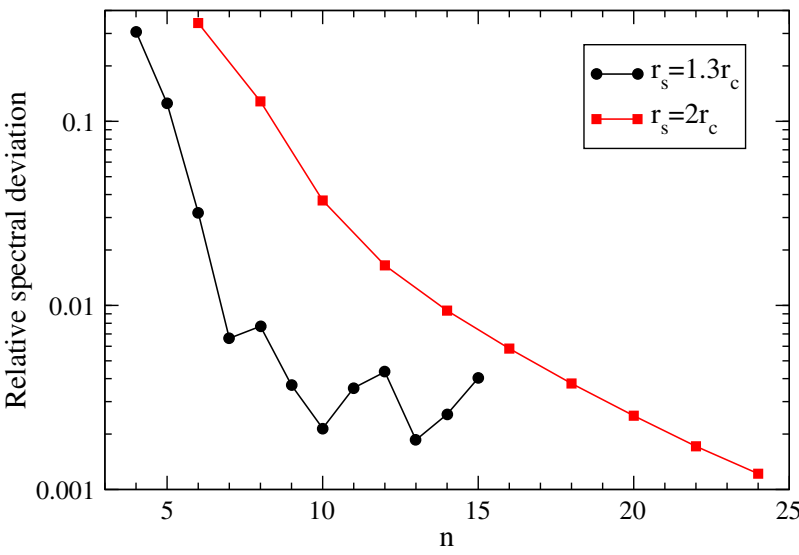

Fig. 4. Integrated absolute differences in total spectral density between OI and GQ calculations as a function of the number of radial gridpoints $n$ used in the GQ calculations. Results are divided by the integral of the total OI spectral density.

for $r_{s}=1.3 r_{c}$ is due to the finite integration radius. On the other hand, the larger value of $r_{s}$ requires higher $n$ values to reach a specific level of accuracy.

To connect back to the $\Delta A_{\text {eff }}$ metric discussed in Subsection 3.A, it can be mentioned that the maximal $\Delta A_{\text {eff }}$ for $n=5$ is $\sim 10 \%$, whereas it is $\sim 1 \%$ for $n=7$. Thus, the accuracy levels achieved for the integrated spectral deviations appear to resemble those found for the effective areas, although a more comprehensive simulation program would be needed to substantiate this as a general claim. As may be appreciated by comparing with Fig. 3, an accuracy level of a few percent in these metrics only implies slight differences in the calculated spectra. Therefore, $r_{s}=1.3 r_{c}$ appears to be an acceptable choice unless in exceptional situations, e.g., if the major part of the input power is coupled into a poorly confined mode. Regarding numerical complexity, the runtime for the GQ method with $n=5$ was similar to that for the overlap integral method. The GQ runtime scales roughly linearly with $n$ as 
expected. Therefore, the OI method is still slightly better than the GQ method for this case of six modes but can be expected to become unfavorable at significantly higher mode numbers.

Of course, the accuracy required of a nonlinear simulation is problem-specific and may in some cases be very high, e.g., if one is interested in modes or frequencies carrying a small part of the total spectral weight. In the present calculations, the small frequency components in the visible spectrum were found to converge with a relative accuracy similar to, or even better than, the spectra around the pump wavelength. In any event, it is an advantage of the GQ approach that numerical convergence can be checked in a straightforward and systematic manner simply by increasing the number of integration points.

\section{Mode Profile Dispersion}

In Section 2, it was described how mode profile dispersion can be accounted for in a straightforward manner using the GQ approach. For the OI approach, on the other hand, a correct treatment in the multimode case is not easy. A systematic approach is to expand the frequency-dependent guided modes in a larger set of frequency-independent basis states, with frequency-dependent expansion coefficients. Even for a singlemode fiber, this is a cumbersome strategy [12] because the numerical complexity increases strongly with the number of basis states used. For the multimode case, using a polynomial expansion as in Refs. [12,13], it was found practically impossible to obtain well-converged results. In particular, the description of modes with cutoffs within the frequency window is challenging with such an expansion. An alternative approach could be to generalize the modified effective area approach described in Refs. [12,19], but the most straightforward generalization to the multimode case will increase the numerical complexity from $O\left(M^{2} N \log (N)\right)$ to $O\left(M^{4} N \log (N)\right)$, which is still a major drawback, and it is difficult to control the approximation in a systematic way. On the other hand, the accuracy of the GQ approach can still be checked simply by increasing $n$ and/or $r_{s}$.

Mode profile dispersion is a particularly important phenomenon for multimode fibers because some modes may have cutoffs appearing within the frequency window of interest. A striking example of this may be appreciated by comparing Fig. 2 with Fig. 5, which shows a similar calculation using the GQ approach with frequency-dependent mode profiles. It can be seen that the inclusion of frequency dispersion suppresses the spectral weight beyond $2 \mu \mathrm{m}$ and also changes the shape of the CR spectrum between 700 and $800 \mathrm{~nm}$. The reason is straightforward: in the calculation without mode profile dispersion, the spectrum beyond $2 \mu \mathrm{m}$ was mainly in the $\mathrm{LP}_{21}$ modes, but these modes (together with the $\mathrm{LP}_{02}$ mode) actually cut off just below $2 \mu \mathrm{m}$ in the fiber under study, meaning that the simulation with fixed mode profiles does not give an accurate description of the nonlinear dynamics, especially since the present calculations do not account for modal losses. In the GQ calculation, the cutoff means that the modes expand strongly, leaving only a little weight in the integration region close to the core; therefore, nonlinear coupling to longer wavelengths in these modes is suppressed, which is physically reasonable. The small spectral weight observed on the $\mathrm{LP}_{21}$ modes just beyond

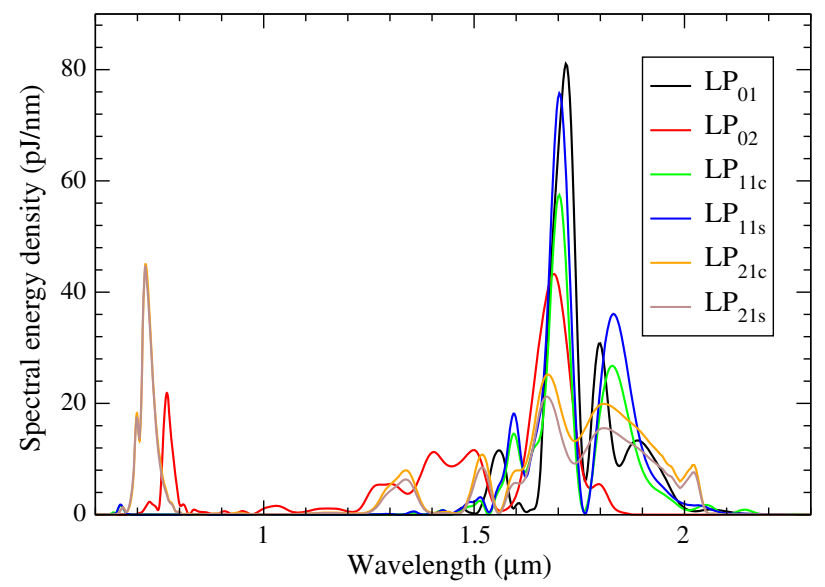

Fig. 5. Final spectral density for the same pulse and fiber parameters as in Fig. 2, when using a GQ approach including mode profile dispersion. $r_{s}=1.3 r_{c}$ and $n=8$.

$2 \mu \mathrm{m}$ appears because the unguided modes still have some fraction of power inside the core due to the boundary condition on the modal field calculations discussed in Subsection 3.A; however, this fraction rapidly goes down with increasing wavelength.

In Fig. 6, the total spectral density, summed over all modes, is plotted for the OI calculation without mode profile dispersion, as well as for GQ calculations with $r_{s}=1.3 r_{c}$, and $n=5-8$ including mode profile dispersion. Apart from showing again the impact of mode profile dispersion, the figure shows that a fair convergence is reached for $n=6$, whereas the curves for $n=7$ and $n=8$ are almost indistinguishable.

To further validate the GQ approach, calculations with $r_{s}=1.3 r_{c}$ and low $n$-values are compared with a reference calculation with $r_{s}=2 r_{c}, n=40$ in Fig. 7, using the same metric as in Fig. 4. An accuracy at the percent level is readily reached already for $n=7$. This indicates that mode profile dispersion does not have a strong impact on GQ convergence, and also that the use of a low $r_{s}$ value is justified, even in the case

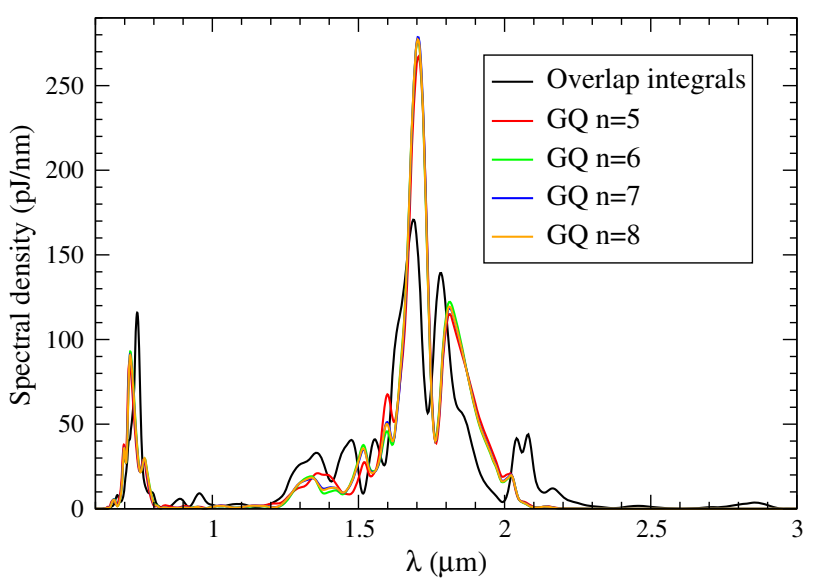

Fig. 6. Total spectral densities, summed over all modes, for the OI calculation without mode profile dispersion shown in Fig. 2, as well as corresponding GQ calculations with $r_{s}=1.3 r_{c}, n=5-8$. 


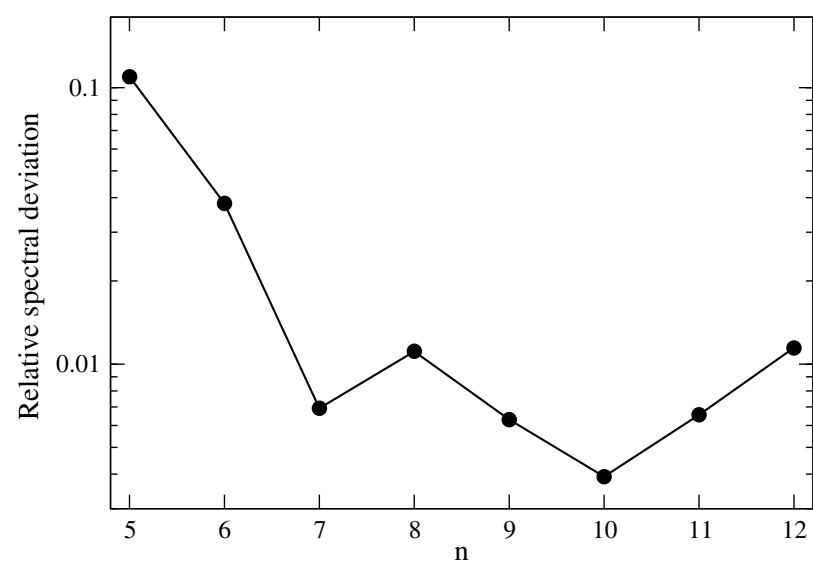

Fig. 7. Integrated absolute differences in total spectral density between a GQ calculation with $r_{s}=2 r_{c}$ and $n=40$ and GQ calculations with $r_{s}=1.3 r_{c}$ as a function of the number of radial gridpoints $n$ used in the latter case. Results are divided by the integral of the total OI spectral density.

where some modes become unguided in the relevant frequency window.

As discussed in Subsection 3.A, the numerical overhead for including mode profile dispersion is a factor of $\sim 2$ for the FFT contribution, whereas there is no change for the terms linear in $N$. In the present example, the computation time was found to increase by $50 \%-60 \%$ when including mode profile dispersion, indicating that both $O(N)$ and $O(N \log N)$ terms contribute significantly to the computational effort.

\section{DISCUSSION}

The GQ method as implemented in this work relies on realspace grids optimized for integrating low-order polynomials, i.e., smooth mode profile distributions. The chosen test case of modes in fibers with weak index contrast is therefore favorable for the method, and further work will be needed to establish its merits in other situations. For waveguides with high index contrasts, such as photonic crystal fibers, or high-index integratedoptics technology, one typically has a situation where both the fields and the value of the nonlinear coefficient change discontinuously over the waveguide cross-section. In such a situation, direct application of a GQ grid is obviously not a good idea. For simple geometries, however, it may be possible to apply the GQ approach by sectioning the nonlinear polarization integral with a GQ grid for each material region. A particularly simple example would be a silica nanofiber, where the method presented in this work could be implemented directly using $r_{s}=r_{c}$, since the nonlinear coefficient of the (air) cladding in this case is negligible [14]. In more complicated geometries, such as solid-core photonic crystal fibers, the choice of a suitable integration grid may be more difficult. A particularly complex example may be hollow-core photonic bandgap fibers, where both silica and air nonlinearity play a significant role, and the silica parts of the fiber have a complex geometry. On the other hand, the more recent class of hollow-core fibers guiding by antiresonant effects in a core with negative-curvature surround features modes with a negligible silica overlap, and a GQ grid confined to the air core may be useful for obtaining nonlinear simulation results [10]. In the absence of ionization effects, this will, of course, only be relevant in fibers where a substantial number of higher order modes are well-confined to the air core with negligible silica overlap, which is perhaps not quite characteristic of current fibers optimized for effective single-mode operation.

As the number of guided modes increases, one must eventually expect the numerical complexity of the method to scale as $O(M N P)$. One can improve this to $O(N P)$ by abandoning the modal expansion entirely [9], evaluating the linear dispersion operator in real space by finite-differences, or possibly $2 \mathrm{D}$ FFTs (in which case one has $O(P N \log N)$ scaling). In this case, however, the dispersion operator is approximated, and $P$ must be chosen large enough to obtain a satisfactory accuracy. The comparison between such methods and the modal GQ integration approach investigated here is therefore not straightforward and is likely to be highly problem-specific.

\section{CONCLUSION}

In conclusion, a numerical approach to nonlinear propagation problems in multimode fibers using on-the-fly real-space integration of the nonlinear polarization on a GQ grid has been investigated. It has been shown that the numerical complexity of this approach scales linearly or at most quadratically with the number of guided modes and that mode profile dispersion may be taken into account with modest computational overhead (except in cases where Raman scattering is neglected). The approach was compared with the widely used approach based on evaluating the nonlinear polarization by summing mode overlap integrals and was found to have a comparable numerical complexity for the particular case of a step-index fiber with six guided modes. Since the overlap integral approach has less favorable scaling properties and is more problematic with respect to inclusion of mode profile dispersion effects, the GQ method will be superior for cases with a large number of guided modes as well as cases where mode profile dispersion is important.

\section{REFERENCES}

1. H. R. Stuart, "Dispersive multiplexing in multimode optical fiber," Science 289, 281-283 (2000).

2. R. Ryf, H. Chen, N. K. Fontaine, A. M. Velazquez-Benitez, J. AntonioLopez, C. Jin, B. Huang, M. Bigot-Astruc, D. Molin, F. Achten, P. Sillard, and R. Amezcua-Correa, "10-mode mode-multiplexed transmission over 125-km single-span multimode fiber," in European Conference on Optical Communication (ECOC) (2015), pp. 1-3.

3. C. Antonelli, M. Shtaif, and A. Mecozzi, "Modeling of nonlinear propagation in space-division multiplexed fiber-optic transmission," J. Lightwave Technol. 34, 36-54 (2016).

4. Y. Ding, J. Xu, H. Ou, and C. Peucheret, "Mode-selective wavelength conversion based on four-wave mixing in a multimode silicon waveguide," Opt. Express 22, 127-135 (2014).

5. J. Demas, P. Steinvurzel, B. Tai, L. Rishoj, Y. Chen, and S. Ramachandran, "Intermodal nonlinear mixing with Bessel beams in optical fiber," Optica 2, 14-17 (2015).

6. S. R. Petersen, T. T. Alkeskjold, C. B. Olausson, and J. Lægsgaard, "Intermodal and cross-polarization four-wave mixing in large-core hybrid photonic crystal fibers," Opt. Express 23, 5954-5971 (2015).

7. L. G. Wright, D. N. Christodoulides, and F. W. Wise, "Controllable spatiotemporal nonlinear effects in multimode fibres," Nat. Photonics $\mathbf{9}$, 306-310 (2015). 
8. L. G. Wright, S. Wabnitz, D. N. Christodoulides, and F. W. Wise, "Ultrabroadband dispersive radiation by spatiotemporal oscillation of multimode waves," Phys. Rev. Lett. 115, 223902 (2015).

9. K. Krupa, A. Tonello, A. Barthélémy, V. Couderc, B. M. Shalaby, A. Bendahmane, G. Millot, and S. Wabnitz, "Observation of geometric parametric instability induced by the periodic spatial self-imaging of multimode waves," Phys. Rev. Lett. 116, 183901 (2016).

10. F. Tani, J. C. Travers, and P. S. Russell, "Multimode ultrafast nonlinear optics in optical waveguides: numerical modeling and experiments in kagome photonic-crystal fiber," J. Opt. Soc. Am. B 31, 311-320 (2014).

11. F. Poletti and P. Horak, "Description of ultrashort pulse propagation in multimode optical fibers," J. Opt. Soc. Am. B 25, 1645-1654 (2008).

12. J. Lægsgaard, "Mode profile dispersion in the generalised nonlinear Schrödinger equation," Opt. Express 15, 16110-16123 (2007).
13. T. X. Tran and F. Biancalana, "An accurate envelope equation for light propagation in photonic nanowires: new nonlinear effects," Opt. Express 17, 17934-17949 (2009).

14. M. Kolesik, E. M. Wright, and J. V. Moloney, "Simulation of femtosecond pulse propagation in sub-micron diameter tapered fibers," Appl. Phys. B 79, 293-300 (2004).

15. N. Akhmediev and M. Karlsson, "Cherenkov radiation emitted by solitons in optical fibers," Phys. Rev. A 51, 2602-2607 (1995).

16. A. Mafi, "Pulse propagation in a short nonlinear graded-index multimode optical fiber," J. Lightwave Technol. 30, 2803-2811 (2012).

17. L. G. Wright, W. H. Renninger, D. N. Christodoulides, and F. W. Wise, "Spatiotemporal dynamics of multimode optical solitons," Opt. Express 23, 3492-3506 (2015).

18. W. H. Press, B. P. Flannery, S. A. Teukolsky, and W. T. Vetterling, Numerical Recipes in Fortran 77 (Cambridge University, 2001).

19. P. V. Mamyshev and S. V. Chernikov, "Ultrashort-pulse propagation in optical fibers," Opt. Lett. 15, 1076-1078 (1990). 